\title{
LA GEOGRAFÍA Y LAS PRÁCTICAS CULTURALES EN LA ENSEÑANZA DEL TURISMO.
}

\author{
Lilia Cristina Beltrán Galindo \\ Maestría en Dirección y Administración de Empresas Turísticas. \\ crisbelt@hotmail.com
}

\section{RESUMEN}

Geografía y Cultura es una suerte de misterioso pleonasmo por descubrir. Porque decir geografía sin decir cultura sería tanto como decir cielo sin decir azul o afirmar el río sin las piedras. La articulación entre geografía, historia material y prácticas culturales ha hecho necesario se desarrolle un interés por la búsqueda de fundamentos científicos, que permitan demostrar tal condición, además de la aplicación y teoría de la geografía humana en las ciencias sociales y la cultura, como complemento fundamental en la enseñanza de la actividad turística; resultando así, ser un componente valioso que permite conocer, fusionarse con un territorio y experimentar un especial recorrido por el mundo. Saber de turismo es entender la cultura que da sentido a la geografía.

El presente artículo busca evidenciar que operar destinos turísticos es entender la cultura que da sentido a la geografía, pues la geografía, como afirmara en su clásico tratado el profesor catalán Pablo Vila, entra por los pies, y está hecha, por tanto, para ser caminada. La enseñanza de la Geografía y Cultura en el turismo es, en ese sentido, una excursión, una caminata, un paseo por el mundo.

PALABRAS CLAVES: Geografía Humana, Cultura, Turismo, Enseñanza. 


\title{
GEOGRAPHY AND CULTURAL PRACTICES IN TOURISM TEACHING \\ Lilia Cristina Beltrán Galindo \\ Maestría en Dirección y Administración de Empresas Turísticas. \\ crisbelt@hotmail.com
}

\begin{abstract}
Geography and Culture are a kind of mysterious undiscovered pleonasm. Saying Geography without saying Culture would be like saying Sky without saying Blue. The relationships between Geography, Material History and Cultural Practices have triggered the development of scientific basis in order to demonstrate such relationships, including the application and theory of human geography in social sciences and culture as an essential complement for teaching tourism. In other words, knowing of tourism implies to understand the culture that gives meaning to geography. This article seeks to evidence that managing tourism destinations involves to understanding the culture that gives sense to geography. In orther words, teaching Geography and Culture in tourism is, in that sense, an excursion, a walk, a trip for the world.
\end{abstract}

KEYWORDS: Human Geography, Culture, Tourism, Education. 


\section{INTRODUCCIÓN}

La reflexión acerca de la importancia de la enseñanza de la Geografía Humana y la Cultura en los programas de turismo, se hace necesaria; siendo este artículo un acercamiento a las teorías de autores que han demostrado el interés por la búsqueda de fundamentos científicos que permitan demostrar la articulación necesaria entre geografía, historia natural y prácticas culturales.

Por lo tanto, entender El "giro cultural" (D. Hiernaux, 2006) de la Geografía que ha permitido la relación con todas las ciencias sociales, dando origen a la Geografía Humana, e identificar los importantes aportes al estudio de la interacción del hombre con su espacio, considerando el espacio su entorno, el territorio donde realiza su asentamiento y los eventos que inciden en su adaptación y desarrollo, es un elemento fundamental para evidenciar cómo trasforma el hombre su espacio y cómo lo afectan los cambios en la naturaleza.

Otro factor a describir, es la cultura como legado y patrimonio de la humanidad y la importancia de conservar y preservar la identidad de los pueblos, hacerlo sostenible en el tiempo con responsabilidad y pertenencia, identificar la cultura como valor agregado de la Geografía Humana (S. Nanda, 1987).

Para finalizar, se analiza la interacción de la geografía humana y la cultura con el turismo. El turismo como valor agregado de la Geografía Humana y la cultura valor agregado del turismo (P. Railey, E. Sierra Valenti, 1981). Una interacción única, que hace obligatoria la enseñanza de estas dos áreas en los programas de turismo.

\section{LA GEOGRAFIA: DEFINICIÓN Y DIVISIONES}

La Real Academia Española indica su origen griego: la geographia, geo (he ge) la-tierra y grafía (graphein) describir. Es la ciencia que trata la descripción de la tierra (Real Academia Española, 2009). La Geografía se interesa en lo físico y humano, así como en las dinámicas (demográficas, socio-económicas, culturales, climáticas, biogeografías, geomorfológicas) que tienen lugar en la Tierra.

Además esta disciplina ha integrado diversos campos culturales como el paisajismo, la literatura descriptiva e inclusive el cine (J. Ortega Valcárcel, 2000), con infinidad de posibilidades en interacción con todas las ciencias sociales.

La Geografía se divide en dos ramas principales, Geografía Física y Geografía Humana. La división se hace solo de manera descriptiva porque al estudiarlas o aplicarlas es 
preciso relacionar ambas ramas (M. Derruau, 1983), teniendo en cuenta que las dinámicas de las sociedades dejan huellas en el medio físico, o la naturaleza viva y cambiante forja cambios en la sociedad que se debe adaptar a ellos.

La Geografía física: es la rama de la geografía que estudia el medio físico. Los principales elementos que le dan estructura al medio físico corresponden al relieve, las aguas terrestres, el clima, la vegetación, la fauna y el suelo; y el estudio de cada uno de estos ha dado origen a diversas ciencias de la Tierra.

La Geografía Humana: es la ciencia social centrada en el estudio de las sociedades y sus territorios; también estudia al ser humano y sus relaciones con su entorno tanto en el aspecto estático de su organización, como en el dinámico de los cambios que experimentan. El giro que ha tenido la Geografía Humana en los últimos 30 años es la resultante de que la complejidad del mundo actual requiera de "acercamientos interdisciplinarios y la construcción de nuevos marcos teóricos que interactúen con otras ciencias sociales" (A. Lindón, D. Hiernaux, 2006) como por ejemplo la geografía de los servicios que estudia las actividades terciarias que se dan en los diferentes espacios. Y la Geografía Turística, que estudia la potencialidad turística de los territorios, los patrones de desarrollo y cambios del turismo, los modelos de desarrollo turístico y los problemas de estos espacios.

\section{GEOGRAFIA HUMANA}

"La Geografía Humana es el estudio de las agrupaciones humanas en sus relaciones con el medio geográfico". (A. Demangeon, 1956). La expresión de medio geográfico es más comprensiva que la de medio físico, abarca no solamente las influencias naturales que pueden ejercerse, sino también una influencia, que contribuye a formar el medio geográfico, el contorno entero, la influencia del mismo hombre.

Cabe anotar, el punto de vista de muchos especialistas que consideran que la Geografía Humana, la cual había perdido interés (o peso) entre las ciencias sociales del siglo XX, hoy se halla en un profundo proceso de renovación gracias al "enfoque cultural" de la Geografía. (D. Hiernaux, 2002).

Por otro lado, la visión moderna de la geografía radica, principalmente, en el cambio que tenemos en el presente de la concepción de lo que es el espacio, la visión global de lo que es la tierra (J. Ortega Valcárcel, 2000), no solamente se ven montañas, ríos, mares, océanos, continentes encontrados, perdidos y por encontrar; hay una visión mucho más importante que es la interacción del hombre con el espacio, con su medio, con su tierra, con su terruño; teniendo en cuenta lo que le aporta la tierra al hombre y la transformación 
que sufre la tierra por el hombre, es decir: una geografía más humana. Nunca observada como antes, limitada a informar, plasmada, estática, sino siempre en movimiento, dinámica, transformadora y renovadora.

Al hacer una reflexión sobre la historia de la geografía, nos damos cuenta de que la historia de la geografía no se separa de la propia historia humana, siendo la cultura su valor agregado más importante. espacio geográfico no es ni sola ni principalmente la naturaleza que lo conforma y lo constituye. El espacio geográfico es en esencia el espacio de los hombres que lo puebla y lo habita, es la cultura de los hombres que ha hecho de ese espacio su país que es lo mismo que decir su paisaje. Porque país y paisaje son parientes muy cercanos.

El geógrafo francés Paul Claval dice: "El actual giro cultural nos hace comprender que la cultura no constituye un sector particular de la vida, sino que desempeña un papel importante en todos los dominios de la Geografía" (1995). Por lo tanto, es imposible separar la cultura de la enseñanza de la Geografía Humana.

\section{LA GEOGRAFIA Y EL TURISMO}

El turismo ha sido analizado esencial y tradicionalmente desde perspectivas estructurales, propias de cierta mirada geográfica. Sin embargo, dos tipos de procesos obligan a repensar esa orientación dominante: las transformaciones mismas de los procesos turísticos, así como la nueva mirada de la Geografía Humana, asociable al "giro cultural". No obstante, la propuesta entonces es hacer hincapié sobre esta nueva mirada, estas constelaciones de cambios que hacen pasar de un turismo visto como un proceso económico estructurante del territorio, a un proceso que construye el espacio a partir de una perspectiva individual y de sus interacciones socio-espaciales. (D.Hiernaux,2008).

Con la premisa de Walter Christaller (1963), "el turismo es esencialmente una actividad económica", la formación académica en el sector turístico se enfocó en sesgar y perfeccionar la visión del turismo como un "producto" más de la economía, teniendo como meta la generación de empleos directos y generación de multiplicadores de los mismos, o la creación, desarrollo y sostenibilidad de centros turísticos.

Este enfoque, por más racional y coherente que pueda parecer en su estructura conceptual, parte de un planteamiento incompleto, dejando por fuera a la Geografía Humana, la cual plantea una premisa que es concluyente: "el acto turístico es un acto individual, ejecutado en un contexto social". Esto permite afirmar que el turismo es un acto societario, porque responde, primero que nada, a toda una serie de procesos que se derivan del funcionamiento mismo de la sociedad. (D. Hiernaux, 1996, 2006). 
El papel preponderante de la actividad turística para la Geografía reside en que esta es capaz de dinamizar el territorio, creando nuevas configuraciones espaciales a partir de la relación hombre-espacio (P. Railey, E. Sierra Valenti, 1981). Pero más allá de esta teoría, resulta interesante distinguir con claridad un turista de un viajero. A formar esa diferencia. El primero, admira y disfruta el espacio natural. El segundo consciente de lo anterior, le suma a eso la comprensión de los espacios culturales que visita, sabe cuál es la particular forma de ser y estar en la geografía del mundo, que tiene esa cultura.

En la actualidad, hablar de turismo es referirse a empresas con actividades muy variadas, desde la agencia de viajes hasta la recreación de un hotel. Es, actualmente, uno de los motores principales de la economía mundial y afecta tanto a países desarrollados como a los países en vía de desarrollo y subdesarrollados. Las actividades que se realizan son muy variadas pues competen tanto a los medios de transporte, como al urbanismo, a la industria de la construcción, a la agricultura, a la ecología, a la cultura, y además ha producido grandes cambios en los paisajes, litorales, montañas, llanos etc. (MA. Troitiño Vinuesa, 1998).

Al mismo tiempo, su peso económico es, en algunas regiones, fundamental y predominante en términos de renta, como de empleo. La OIT (Organización Internacional del Trabajo) estima en 200 millones de personas en todo el mundo las que trabajan en el sector de viajes y turismo, lo que equivale al $8 \%$ del empleo mundial. Así mismo, señala que cada puesto de trabajo genera aproximadamente otro puesto y medio (indirecto) en actividades económicas relacionadas con él. (Hiernaux 2006).

Ahora bien, el gran número de empleos que genera la industria turística y el impacto que produce en el territorio permiten calibrar la gran importancia que está adquiriendo en el mundo actual. El crecimiento del turismo puede volverse problemático en muchos casos, ya que puede llegar a ser excesivo. La oferta turística es cada vez mayor en los países menos desarrollados. Cada vez son más numerosos los viajes a estos destinos. Se debe tener mucho cuidado en el respeto por el medio ambiente, en no caer en el capitalismo salvaje y la conservación de las características físicas, culturales, ambientales, sociológicas, que hacen deseables estos destinos, si no se quiere romper el equilibrio en la demanda turística (P. Claval, 1999).

En síntesis, siendo el territorio el principal objeto de estudio de la geografía, surge la importancia de la actividad turística como valor agregado a un territorio, ya sea en lo social, económico, ambiental, cultural, paisajístico, etc.. La importancia de la actividad turística para la geografía, reside en que esta es capaz de dinamizar el territorio, creando nuevas configuraciones espaciales a partir de la relación hombre-espacio. (P. Railey, E. Sierra Valenti, 1981). 


\section{LA ENSEÑANZA DE LA GEOGRAFÍA EN EL TURISMO.}

Llegado a este punto, resaltar la importancia de la enseñanza de la Geografía Humana en turismo radica en que se debe tener "un conocimiento básico del espacio y la relación del hombre con el espacio" (B. Mérenne-Schoumaker, 2006) su desarrollo, interacción, proyección, sostenibilidad de los proyectos, entender y explicar las reglas de funcionamiento de los diferentes territorios, de las sociedades humanas en el seno de esos espacios, los hechos que conllevan a una organización social, su cultura y entender y explicar las dinámicas de los cambios en los asentamientos.

Cabe señalar que adoptar un razonamiento geográfico es fundamental. Es, sin duda, uno de los pilares de la geografía actual y constituye, centrándola alrededor de los problemas territoriales y ambientales, uno de los caminos para vertebrar el proyecto geográfico del conocimiento. Una perspectiva integradora, centrada en el análisis de las dinámicas del espacio humanizado y orientada a territorializar la Geografía Humana, puede permitir superar las limitaciones operativas del razonamiento geográfico y darle una capacidad propositiva. (MA. Troitiño Vinuesa, 2009).

Aquí vale la pena hacer una pequeña digresión sobre el método de estudios seguido por los geógrafos, afirmando que es similar al de otras disciplinas: se empieza con la observación y la subsiguiente recolección de informaciones y datos. El trabajo de campo (reconocimiento del terreno, encuestas y entrevistas, mediciones y grabaciones, etc.), los experimentos de laboratorio, o las búsquedas en archivos son, entre otros, procesos habituales para reunir información sobre las relaciones geográficas. Una vez los datos han sido recogidos, el siguiente paso es la visualización o representación (que puede incluir descripciones escritas, mapas, diagramas, tablas, fórmulas matemáticas, etc.) y que permite que la información pueda ser explorada, sintetizada y ofrecida a otros. En la parte central está el análisis, ya sea cuantitativo o cualitativo, que servirá para descubrir principios y regularidades y formular relaciones e hipótesis. (B. Mérenne-Schoumaker, 2006).

De igual modo, los instrumentos y metodologías de los geógrafos también son similares a los usados por científicos sociales y de las humanidades. Hay una herramienta que distingue y caracteriza el trabajo del geógrafo: el mapa. Sirve de información y de análisis. Avanzando en el tiempo encontramos que el Sistema de Información Geográfica (SIG) se ha convertido en uno de los métodos más importantes para el análisis geográfico (L. Sánchez Ayala, 2012). Un SIG es una colección organizada de programas informáticos y datos geográficos que ha sido diseñada para capturar, almacenar, actualizar, manipular y mostrar información geográficamente referenciada, de gran apoyo para el estudio de la geografía. 
Así empezamos a cercar, pues, el importante enfoque en la enseñanza del turismo con competencias transversales de cartografía, desplazamientos de la población sin soslayar los problemas y el impacto de los fenómenos de la naturaleza en los paisajes y regiones, identificando así la posibilidad y sostenibilidad de los proyectos turísticos. (Autor: B. Mérenne-Schoumaker, 2006). Este enfoque permitirá formar visionarios en destinos y posibilidades turísticas, siendo profesionales íntegros y conocedores del entorno, su historia geográfica y la huella dejada por el hombre en el espacio, entendiendo que un espacio geográfico no es ni sola ni principalmente la naturaleza que lo conforma y lo constituye.

\section{LA CULTURA}

Por otro lado, los individuos y los grupos están condicionados por la educación que han recibido: la cultura aparece así como una herencia. Las modalidades según las cuales se transmite de una generación a la otra o de un lugar a otro, en virtud de los intercambios, los desplazamientos de corta duración o las migraciones, dependen del medio y del nivel técnico y contribuyen ampliamente a la diversidad de las sociedades (L. Sánchez Ayala, 2012).

Cierto es que, la cultura es el conjunto aprendido de tradiciones y estilos de vida, socialmente adquiridos, de los miembros de una sociedad, incluyendo sus modos pautados y repetitivos de pensar, sentir y actuar (es decir, su conducta). (S. Nanda, 1982).

Bien pareciera por todo lo anterior, que la cultura es un conjunto de ideas, creencias y valores aprendidos y aceptados por un grupo de personas (S. Nanda, 1980). Estas ideas, creencias y valores se pueden ver reflejados en códigos, símbolos, normas de comportamiento social o en instituciones sociales como la familia y la religión. Pero la cultura es influenciable al espacio, o sea que cambia de acuerdo con los espacios y lugares. Por lo tanto, existen variaciones culturales tales como las subculturas, o grupos de personas que pertenecen a un grupo cultural mayor y presentan características que de alguna forma los diferencian del resto del grupo cultural. Este conjunto de elementos culturales forman subculturas, pero la gran variedad de lenguas, la diversidad de etnias, sus mezclas y religiones hacen divisiones culturales aún más grandes y radicales. (G. Yúdice, 2002).

Hay otro aspecto que se debe tener en cuenta y es que la cultura cuenta tanto con componentes materiales como no materiales. La cultura no material consiste en aspectos intangibles como creencias, ideales, valores o patrones de conducta que son expresados y a la vez plasmados mediante la cultura material.

La cultura material incluye una extensa y diversa gama de artículos, por lo que la cultura 
material no existe independientemente de la cultura no material. Los productos culturales desempeñan un papel importante en el estudio de culturas del pasado, como es identificar o aprender de las actividades diarias, patrones alimenticios, nivel tecnológico, prácticas religiosas o sistemas de valoración de culturas pasadas.

El patrimonio cultural de un pueblo o región se puede dividir en dos: Patrimonio material, constituido por los lugares consagrados a la cultura, realizaciones hechas por el hombre, museos, monumentos, pueblos y ciudades artísticas (con relevancia arquitectónica), patrimonio arqueológico y parques prehistóricos, jardines, edificios religiosos, militares, etc. Y el Patrimonio inmaterial o intangible como fiestas y festivales, encuentros, tradiciones y maneras de hacer donde el pasado y el presente se hacen presentes. (P. Claval, 1999).

En este punto, es prudente advertir que, el patrimonio cultural, nos atañe a todos, es responsabilidades de todos. El reto es encontrar estrategias y políticas en las que se asuma esta responsabilidad, que contribuyan todos a su conservación (B Mérenne-Schoumaker, 2006).

\section{LA CULTURA Y EL TURISMO}

El papel de la cultura se ha expandido de una manera sin precedentes al ámbito político y económico por la rápida globalización, considerándola como un "recurso". El uso creciente de la cultura para el mejoramiento, tanto sociopolítico como económico, ha repercutido, en la participación progresiva, conflictos sobre la ciudadanía y el resurgimiento del capitalismo cultural (J. Rifkin, 2002). Lo anterior parece confirmar que la cultura además de ser considerada como un incentivo para el crecimiento económico, permite la consolidación de una ciudadanía fundada en la participación activa de la población. Todo esto lleva a suponer que la cultura es un motor del desarrollo capitalista. Cabe señalar que la identidad cultural de un pueblo viene definida históricamente a través de múltiples aspectos en los que se plasma su cultura; como la lengua, instrumento de comunicación entre los miembros de una comunidad, las relaciones sociales, ritos y ceremonias propias, o los comportamientos colectivos, es decir, los sistemas de valores y creencias. Un rasgo propio de estos elementos de identidad cultural es su carácter inmaterial y anónimo, pues son producto de la colectividad (S. Nanda, 1987). Para ilustrar mejor podemos tener en cuenta que el cine y la música son fundamentales para la identidad cultural.

Todo esto parece confirmar que es prioridad del turismo promover la cultura, ser propagadores, multiplicadores y voceros del legado cultural de los ancestros, preservando la identidad cultural de las sociedades y protegiendo y conservando el patrimonio 
cultural, material y no material de la humanidad, a través de la interacción de los hombres sobre su territorio. Y territorio viene de tierra pero no se reduce a ella. El territorio es una construcción. La cultura, es su motor, su aliento. Y el acercamiento para entender la transformación de los espacios naturales en espacios culturales de todo orden, es nuestro objetivo más preciado. Saber de turismo es entender la cultura que da sentido a la geografía.

Sin duda alguna, la cultura aparece pues como una herencia, se transmite además de una generación a otra o de un lugar a otro, en virtud de los intercambios, los desplazamientos de corta duración o las migraciones, que contribuyen ampliamente a la diversidad cultural de las sociedades (D. Hiernaux, A. Lindón, 2006).

Las anteriores consideraciones fundamentan que la cultura como herencia, identidad de los pueblos, motor de la economía, patrimonio físico y cultural de la humanidad y transformadora y renovadora de los espacios, no puede pasar inadvertida en los programas educativos de las escuelas de turismo. Representa una parte fundamental para entender el proceso, desarrollo de los espacios y además, permite a los individuos y a los grupos proyectarse en el futuro y en otros mundos diversos (G. Yudice, 2002).

Ahora bien, no se debe soslayar que el turismo cultural, "basado en las atracciones culturales que posee el destino, ya sean permanentes o temporales, [...] o basado en las características culturales y/o sociales de una población que dispone de un estilo tradicional de vida o de unas características propias" (OMT, 1998), ofrece un doble beneficio desde una perspectiva social y del patrimonio histórico; por un lado como herramienta de defensa de la tradición y por otro lado como vehículo de la democratización cultural, avance social y adquisición de derechos de la tercera generación (también conocidos como derechos de solidaridad o de los pueblos) (M.Ramos, 2007), adicionalmente juega un papel importante en aspectos como la recuperación y revalorización del patrimonio y el fortalecimiento de identidades.

En este mismo orden de ideas, la UNESCO, con el objetivo de contribuir a la lucha contra la pobreza, a la defensa del medio ambiente y a un aprecio mutuo de las culturas, pone de manifiesto la necesidad de formular políticas que tengan en cuenta la relación entre el turismo y diversidad cultural, diálogo intercultural, y desarrollo (UNESCO, 2008); resaltando así la importancia de la esfera cultural en el turismo como motor de cambio social y desarrollo sostenible. De este modo el turismo cultural aparece como una herramienta que puede contribuir al alcance de estos objetivos; sin embargo es vital tener en cuenta que el consumo de bienes culturales en sí mismo no conduce automáticamente al cambio social (M. Ramos, 2007), sin el desarrollo de políticas y estrategias que enmarquen este mercado cultural, lo que necesariamente incluye planes académicos en el sector turístico que contribuyan a un real conocimiento de la cultura y su evolución. 
El fomento de la cultura en articulación con la cadena de valor del turismo, genera un instrumento para el desarrollo sostenible, el dialogo intercultural y la cultura de paz, desarrollando de este modo un importante impacto social.

\section{CONCLUSIONES}

Para generar conocimiento en la operación de la actividad turística, resulta ser importante el estudio de la geografía, paralelo a las características culturales de las regiones, además de la incidencia sobre la población y sus costumbres, siendo esta parte fundamental de la formación del futuro profesional, buscando así generar una competencia con la que se identifique, valore y ofrezca la oferta turística y cultural de una región.

Es necesario recalcar que para la operación del turismo es necesario entender la Geografía Humana como una herramienta de conocimiento, razonando y comprendiendo el desarrollo y la interacción del hombre con su espacio. Saber, entender y explicar las reglas de funcionamiento de los diferentes territorios, de las sociedades humanas en el seno de sus espacios, considerar los hechos que conllevan a una organización social determinada, estudiar, los desplazamientos, asentamientos, las dinámicas de los cambios, para formar un modo de pensamiento geográfico, que ayude a identificar, promover y proyectar la sostenibilidad de los lugares con vocación turística.

La tesis que ahora voy a expresar es que los operadores del turismo deben conocer a fondo la cultura como lo que es: "un valor agregado" del turismo. Pero la cultura en sus raíces, como "herencia" de civilizaciones antiguas y de los nuestros ancestros; conociendo a fondo sus creencias, costumbres, tradiciones que hacen especial un sitio turístico. Saber el porqué de las transformaciones sociales. Ayudar a mantener y preservar los valores culturales materiales y no-materiales de un grupo social, como un preciado "legado" de la nuestra historia. Utilizar la cultura como una herramienta, ser voceros y multiplicadores del "gran legado".

\section{BIBLIOGRAFIA}

Albet, A .Benejam, P. (2006). Una Geografía Humana Renovada. (1² Edición). España: Ediciones Vicens-Vives.

Claval, P. (1995). La Geografía Cultural. (1 Edición). Francia: Ediciones Nathan.

Claval, P. (2002). El enfoque Cultural y las Concepciones Geográficas del Espacio. Boletín de la Asociación de Geógrafos Españoles. (N³4). 21-39.

Demangeon, A. (1956). Problemas de la Geografía Humana. ( $1^{\circ}$ Edición). Barcelona: Ediciones Omega S.A.

Derruau, M. (1983). Geografía Humana. ( $1^{\circ}$ Edición). España: Vicens Vives.

Hiernaux D. (2008). Una década de cambios: La Geografía Humana y el estudio del 
Turismo. Revista Electrónica de Ciencias Sociales. Vol.XII (N²70), 1-9.

Hiernaux, D. y Lindón, A. (2006). Tratado de Geografía Humana. En F. Fernández Christlieb. (Ed.), Geografía Cultural (pp.220-252). México: Anthropos.

Hiernaux, D. y Lindón, A. (2006). Tratado de Geografía Humana. En D. Hiernaux. (Ed.), Geografía del Turismo (pp.401-432). México: Anthropos.

Hiernaux, D. y Lindón, A. (2006). Tratado de Geografía Humana. En B. MérenneSchoumaker. (Ed.), La Enseñanza de la Geografía. (pp.628-642). México: Anthropos.

Hiernaux, D. y Lindón, A. (2006). Tratado de Geografía Humana. En Lindón, A., (Ed.), Geografías de la vida cotidiana (pp.356-399). México: Anthropos.

Jones, E. (1966). Geografía Humana. ( $1^{\circ}$ Edition). Londres: Chatto and Windus.

Medus, NB. (1997). Los Estudios Electorales en la Geografía. ( $1^{\circ}$ Edición). Argentina: Huellas.

Nanda, S. (1980). Antropología Cultural Adaptaciones Socioculturales. ( $1^{\circ}$ Edición). México: Grupo Editorial Iberoamérica.

Nanda, S. (1980). Antropología Cultural Adaptaciones Socioculturales. En S. Nanda (Ed.) El Concepto de Cultura. (pp. 39-52). México: Grupo Editorial Iberoamérica.

Ortega Valcárcel, J. (2000.) Los Horizontes de la Geografía.(1² Edición) España: Ariel S.A.

Railey, P., Sierra Valenti, E. (1981). La Didáctica de la Geografía: diez años de evolución. España: Editorial Cincel.

Romero González, J. (2007). Geografía Humana: Procesos, riesgos, incertidumbres en un mundo globalizado. ( $2^{\circ}$ Edición). España: Ariel.

Sánchez Ayala, L. (2012). Geografía Humana Conceptos Básicos y Aplicaciones. (1 Edición). Colombia: Ediciones Uniandes.

Troitiño Vinuesa, MA. (1992). Dimensión Aplicada y Utilidad social de la Geografía Humana. Revista Cuatrimestral de Geografía. Ería. Revistas de la Universidad de Oviedo. (N²7) 57-73.

Yudice, G. (2003). Los estudios culturales en la encrucijada de la incertidumbre. Revista Iberoamericana. Vol. LXIX ( $\left.{ }^{\circ} 203\right), 449-464$. 